\title{
Bell inequalities for three particles
}

\author{
Jing-Ling Chen, ${ }^{1,2, *}$ Chunfeng $\mathrm{Wu},{ }^{2,3}$ L. C. Kwek, ${ }^{2,4}$ and C. H. $\mathrm{Oh}^{2, \dagger}$ \\ ${ }^{1}$ Theoretical Physics Division, Chern Institute of Mathematics, \\ Nankai University, Tianjin 300071, People's Republic of China \\ ${ }^{2}$ Department of Physics, National University of Singapore, 2 Science Drive 3, Singapore 117542 \\ ${ }^{3}$ School of Physics, Northeast Normal University, Changchun 130024, People's Republic of China \\ ${ }^{4}$ Nanyang Technological University, National Institute of Education, 1, Nanyang Walk, Singapore 637616
}

We present new tight Bell inequalities expressed by probabilities for three four- and fivedimensional systems. The tight structure of Bell inequalities for three $d$-dimensional systems (qudits) is proposed. Some interesting Bell inequalities of three qubits reduced from those of three qudits are also studied.

PACS numbers: 03.65.Ud, 03.67.-a, 42.50.-p

\section{INTRODUCTION}

That no local and realistic theory agrees with all predictions of quantum mechanics was shown by Bell in 1964 [1] through the violations of certain constraints. Local realism imposes constraints in the form of Bell inequalities on statistical correlations of measurements on multiparticles. Quantum mechanics predicts violations of such Bell inequalities. The original Bell inequality and the subsequent famous CHSH inequality [2], the latter being cast into a form more amenable for experimental verification, were formulated for the simplest composite quantum system, namely, a system of two qubits.

Bell inequalities which eliminate local realistic description are of importance not only on fundamental research but on identifying ultimate resources for quantum information processing. It was shown that there is a direct link between the security of quantum cryptography and the violation of Bell inequalities [3, 4]. Collins et al [5] found a tight Bell inequality for two arbitrary $d$-dimensional systems (or two qudits) in terms of joint probabilities, hereafter we call it as the CGLMP inequality. For $d=2$, the CGLMP inequality reduces to the CHSH inequality. Alternatively, for $N$ particles of two dimensions (called $N$-qubit), it was shown that there exists a general Bell inequality which is a sufficient and necessary condition for $N$-body correlations to be describable in a local and realistic theory based on two local settings for each observer [ [6]. However, Bell inequalities for $N(N>2)$ entangled $d$-dimensional $(d>2)$ quantum systems are not so well formulated as those for two-qudit or $N$-qubit. Only recently, the problems have been solved partly in the case of three three-dimensional particles in [7]. The authors developed a coincidence Bell inequality in terms of probabilities. For general $N(N>2)$ entangled $d$-dimensional quantum systems, no Bell inequality for either probabilities or correlation functions has been presented until now, although GHZ paradox has been generalized to $N$ qudits systems [8].

Even for $N$-qubit systems, there is one problem on Bell inequality remains, that is "do all pure entangled states violate Bell inequalities for correlation functions"? In other words, it is that whether the theorem of Gisin [9, 10] can be generalized to $N$ qubits or not. It is found that there is a family of pure entangled states of $N$ qubits which do not violate all Bell inequalities [11]. For three qubits, we have proposed a Bell inequality to solve the problem [12].

In this work, we present the tight Bell inequalities expressed by probabilities for three four- and five-dimensional systems. The tight structure of Bell inequalities for three $d$-dimensional systems (qudits) is proposed. Some interesting Bell inequalities for three qubits reduced from those of three qudits are also studied.

\section{BELL INEQUALITIES FOR THREE $d$-LEVEL SYSTEMS}

Local realism cannot exhibit arbitrary correlations. The constraints that local realistic correlations must obey can be written in the form of Bell inequalities. For a three $d$-dimensional system with an arbitrary value of $d$, some efforts have been given to develop Bell inequalities recently. The first step came in 1990 with a paper of Mermin [13] in which

*Electronic address: chenjl@nankai.edu.cn

†Electronic address: phyohch@nus.edu.sg 
he derived a Bell inequality for arbitrary $N$-qubit states; quantum mechanics violates this inequality by an amount that grows with $N$. This result clearly gives us a first three-qubit Bell inequality in a correlation form,

$$
Q_{112}+Q_{121}+Q_{211}-Q_{222} \leq 2
$$

It can be expressed in terms of probabilities,

$$
\begin{aligned}
& P\left(a_{1}+b_{1}+c_{2}=0\right)-P\left(a_{1}+b_{1}+c_{2}=1\right)+P\left(a_{1}+b_{2}+c_{1}=0\right)-P\left(a_{1}+b_{2}+c_{1}=1\right) \\
& +P\left(a_{2}+b_{1}+c_{1}=0\right)-P\left(a_{2}+b_{1}+c_{1}=1\right)-P\left(a_{2}+b_{2}+c_{2}=0\right)+P\left(a_{2}+b_{2}+c_{2}=1\right) \leq 2 .
\end{aligned}
$$

This Bell inequality is maximally violated by the three-qubit GHZ state $\left|\psi_{2}\right\rangle=\frac{1}{\sqrt{2}}(|000\rangle+|111\rangle)$. But for the generalized GHZ states $\left|\psi_{2}\right\rangle_{G H Z}=\cos \xi|000\rangle+\sin \xi|111\rangle$, there exists one region $\xi \in(0, \pi / 12]$ in which the Bell inequality is not violated.

The second step is due to Ref. [7]. The authors developed a three-qutrit Bell inequality involving probabilities which can be given in an alternative form,

$$
\begin{aligned}
& -P\left(a_{1}+b_{1}+c_{1}=0\right)-P\left(a_{1}+b_{1}+c_{1}=1\right)+2 P\left(a_{1}+b_{1}+c_{1}=2\right)+P\left(a_{1}+b_{1}+c_{2}=0\right) \\
& -2 P\left(a_{1}+b_{1}+c_{2}=1\right)+P\left(a_{1}+b_{1}+c_{2}=2\right)+P\left(a_{1}+b_{2}+c_{1}=0\right)-2 P\left(a_{1}+b_{2}+c_{1}=1\right) \\
& +P\left(a_{1}+b_{2}+c_{1}=2\right)+P\left(a_{2}+b_{1}+c_{1}=0\right)-2 P\left(a_{2}+b_{1}+c_{1}=1\right)+P\left(a_{2}+b_{1}+c_{1}=2\right) \\
& +2 P\left(a_{1}+b_{2}+c_{2}=0\right)-P\left(a_{1}+b_{2}+c_{2}=1\right)-P\left(a_{1}+b_{2}+c_{2}=2\right)+2 P\left(a_{2}+b_{1}+c_{2}=0\right) \\
& -P\left(a_{2}+b_{1}+c_{2}=1\right)-P\left(a_{2}+b_{1}+c_{2}=2\right)+2 P\left(a_{2}+b_{2}+c_{1}=0\right)-P\left(a_{2}+b_{2}+c_{1}=1\right) \\
& -P\left(a_{2}+b_{2}+c_{1}=2\right)-2 P\left(a_{2}+b_{2}+c_{2}=0\right)-2 P\left(a_{2}+b_{2}+c_{2}=1\right)+4 P\left(a_{2}+b_{2}+c_{2}=2\right) \leq 6 .
\end{aligned}
$$

The above inequality is maximally violated by the three-qutrit GHZ state $\left|\psi_{3}\right\rangle=\frac{1}{\sqrt{3}}(|000\rangle+|111\rangle+|222\rangle)$. It is worthy of mentioning that both inequalities (2) and (3) are tight.

Here the third step is coming. Our approach of constructing a new Bell inequality for tripartite four-dimensional systems is based on the Gedanken experiment. There are three separated observers, denoted by $\mathrm{A}, \mathrm{B}$, and $\mathrm{C}$ hereafter, each can carry out two possible local measurements, $A_{1}$ or $A_{2}$ for $\mathrm{A}, B_{1}$ or $B_{2}$ for $\mathrm{B}$ and $C_{1}$ or $C_{2}$ for C respectively. Each measurement may have four possible outcomes, labeled by $0,1,2$ and 3 . We denote the observable $X_{i}$ measured by party $X$ and the outcome $x_{i}$ with $X=A, B, C(x=a, b, c)$. A local realistic theory can be described by $8 \times 56$ probabilities. Here we denote the joint probability $P\left(a_{i}+b_{j}+c_{k}=r\right)$ that the measurements $A_{i}, B_{j}$ and $C_{k}$ have outcomes that differ, modulo four, by $r$ :

$$
P\left(a_{i}+b_{j}+c_{k}=r\right)=\sum_{a, b=0,1,2,3} P\left(a_{i}=a, b_{j}=b, c_{k}=r-a-b\right) .
$$

Some of the local realistic constraints are trivial, such as normalization and the no-signaling conditions which are not violated by quantum predictions. Only the non-trivial inequality, which is not true for quantum mechanics, is of use for checking whether we can describe quantum correlations by a classical model. The new Bell inequality for three four-dimensional systems reads

$$
\begin{aligned}
& -5 P\left(a_{1}+b_{1}+c_{1}=0\right)+P\left(a_{1}+b_{1}+c_{1}=1\right)+3 P\left(a_{1}+b_{1}+c_{1}=2\right)+P\left(a_{1}+b_{1}+c_{1}=3\right) \\
& +3 P\left(a_{1}+b_{1}+c_{2}=0\right)-7 P\left(a_{1}+b_{1}+c_{2}=1\right)+3 P\left(a_{1}+b_{1}+c_{2}=2\right)+P\left(a_{1}+b_{1}+c_{2}=3\right) \\
& +3 P\left(a_{1}+b_{2}+c_{1}=0\right)-7 P\left(a_{1}+b_{2}+c_{1}=1\right)+3 P\left(a_{1}+b_{2}+c_{1}=2\right)+P\left(a_{1}+b_{2}+c_{1}=3\right) \\
& +3 P\left(a_{2}+b_{1}+c_{1}=0\right)-7 P\left(a_{2}+b_{1}+c_{1}=1\right)+3 P\left(a_{2}+b_{1}+c_{1}=2\right)+P\left(a_{2}+b_{1}+c_{1}=3\right) \\
& +3 P\left(a_{1}+b_{2}+c_{2}=0\right)+P\left(a_{1}+b_{2}+c_{2}=1\right)-5 P\left(a_{1}+b_{2}+c_{2}=2\right)+P\left(a_{1}+b_{2}+c_{2}=3\right) \\
& +3 P\left(a_{2}+b_{1}+c_{2}=0\right)+P\left(a_{2}+b_{1}+c_{2}=1\right)-5 P\left(a_{2}+b_{1}+c_{2}=2\right)+P\left(a_{2}+b_{1}+c_{2}=3\right) \\
& +3 P\left(a_{2}+b_{2}+c_{1}=0\right)+P\left(a_{2}+b_{2}+c_{1}=1\right)-5 P\left(a_{2}+b_{2}+c_{1}=2\right)+P\left(a_{2}+b_{2}+c_{1}=3\right) \\
& \left.-P\left(a_{2}+b_{2}+c_{2}=0\right)-3 P\left(a_{2}+b_{2}+c_{2}=1\right)-P\left(a_{2}+b_{2}+c_{2}=2\right)+5 P\left(a_{2}+b_{2}+c_{2}=3\right)\right) \leq 12 .
\end{aligned}
$$

That the maximum value of the left hand side of inequality (5) for local theories is 12 can be given in the following sense. By using $\sum_{r=0}^{3} P\left(a_{i}+b_{j}+c_{k}=r\right)=1$, the inequality (5) is reformed as

$$
\begin{aligned}
& -3 P\left(a_{1}+b_{1}+c_{1}=0\right)+P\left(a_{1}+b_{1}+c_{1}=2\right)-5 P\left(a_{1}+b_{1}+c_{2}=1\right) \\
& -P\left(a_{1}+b_{1}+c_{2}=3\right)-5 P\left(a_{1}+b_{2}+c_{1}=1\right)-P\left(a_{1}+b_{2}+c_{1}=3\right) \\
& -5 P\left(a_{2}+b_{1}+c_{1}=1\right)-P\left(a_{2}+b_{1}+c_{1}=3\right)+P\left(a_{1}+b_{2}+c_{2}=0\right) \\
& -3 P\left(a_{1}+b_{2}+c_{2}=2\right)+P\left(a_{2}+b_{1}+c_{2}=0\right)-3 P\left(a_{2}+b_{1}+c_{2}=2\right) \\
& +P\left(a_{2}+b_{2}+c_{1}=0\right)-3 P\left(a_{2}+b_{2}+c_{1}=2\right)-P\left(a_{2}+b_{2}+c_{2}=1\right) \\
& \left.+3 P\left(a_{2}+b_{2}+c_{2}=3\right)\right) \leq 0 .
\end{aligned}
$$


To beat the bound 0 , terms $P\left(a_{1}+b_{1}+c_{1}=2\right)$ and $P\left(a_{2}+b_{2}+c_{2}=3\right)$ are taken equal to one first. This means that $a_{1}+b_{1}+c_{1}+a_{2}+b_{2}+c_{2}=5$. Among the remained terms, we take $P\left(a_{1}+b_{1}+c_{2}=3\right), P\left(a_{1}+b_{2}+c_{1}=3\right)$ and $P\left(a_{2}+b_{1}+c_{1}=3\right)$ equal to one to maximize the value of left hand side of the inequality (6). As a result, $a_{2}+b_{2}+c_{1}=2, a_{2}+b_{1}+c_{2}=2$ and $a_{1}+b_{2}+c_{2}=2$ according to the constraint $a_{1}+b_{1}+c_{1}+a_{2}+b_{2}+c_{2}=5$. So $P\left(a_{1}+b_{1}+c_{2}=1\right)=P\left(a_{1}+b_{2}+c_{1}=1\right)=P\left(a_{2}+b_{1}+c_{1}=1\right)=0, P\left(a_{2}+b_{2}+c_{1}=2\right)=P\left(a_{2}+b_{1}+c_{2}=2\right)=$ $P\left(a_{1}+b_{2}+c_{2}=2\right)=1$ and $P\left(a_{2}+b_{2}+c_{1}=0\right)=P\left(a_{2}+b_{1}+c_{2}=0\right)=P\left(a_{1}+b_{2}+c_{2}=0\right)=0$. Therefore we have $0+1-0-1-0-1-0-1+0-3+0-3+0-3-0+3=-8 \leq 0$. If initially terms $P\left(a_{1}+b_{1}+c_{1}=0\right)$ and $P\left(a_{2}+b_{2}+c_{2}=3\right)$ are taken equal to one first. This means that $a_{1}+b_{1}+c_{1}+a_{2}+b_{2}+c_{2}=3$. Among the remained terms, we take $P\left(a_{1}+b_{1}+c_{2}=3\right), P\left(a_{1}+b_{2}+c_{1}=3\right)$ and $P\left(a_{2}+b_{1}+c_{1}=3\right)$ equal to one to maximize the value of left hand side of the inequality (6). As a result, $a_{2}+b_{2}+c_{1}=0, a_{2}+b_{1}+c_{2}=0$ and $a_{1}+b_{2}+c_{2}=0$ according to the constraint $a_{1}+b_{1}+c_{1}+a_{2}+b_{2}+c_{2}=3$. So $P\left(a_{1}+b_{1}+c_{2}=1\right)=P\left(a_{1}+b_{2}+c_{1}=1\right)=P\left(a_{2}+b_{1}+c_{1}=1\right)=0$, $P\left(a_{2}+b_{2}+c_{1}=2\right)=P\left(a_{2}+b_{1}+c_{2}=2\right)=P\left(a_{1}+b_{2}+c_{2}=2\right)=0$ and $P\left(a_{2}+b_{2}+c_{1}=0\right)=P\left(a_{2}+b_{1}+c_{2}=0\right)=$ $P\left(a_{1}+b_{2}+c_{2}=0\right)=1$. Therefore we have $-3+0-0-1-0-1-0-1+1-0+1-0+1-0-0+3=0 \leq 0$. Therefore, after some simple and patient calculations, it can be shown that the inequality (6) is always bounded by 0 in a local realistic model. Furthermore, the Bell inequality (5) is a tight inequality for three four-dimensional systems [14].

Let us now consider the maximum value that can be attained for the inequality (5) for quantum measurements on an entangled quantum state. First, we specify the quantum state and measurement. The initial state is a natural generalization of bipartite maximally entangled state to three four-level systems,

$$
\left|\psi_{4}\right\rangle=\frac{1}{2}(|000\rangle+|111\rangle+|222\rangle+|333\rangle) .
$$

Consider a Gedanken experiment in which A, B and C measure observables defined by unbiased symmetric multi-port beam splitters [15] on $|\psi\rangle$. The unbiased symmetric multi-port beam splitter is an optical device with $d$ input and $d$ output ports. In front of every input port there is a phase shifter that changes the phase of the photon entering the given port. If a phase shifter in some input port is set to zero and a photon enters the device through this port then it has an equal chance of leaving the device through any output port. The phase shifters can be changed by the observers; they represent the local macroscopic parameters available to the observers. The matrix elements of an unbiased symmetric multi-port beam splitter are given by $U_{k l}(\vec{\phi})=\frac{1}{\sqrt{d}} \alpha^{k l} \exp \left(i \phi^{l}\right)$, where $\alpha=\exp \left(\frac{2 i \pi}{d}\right)$ and $\phi^{l}(l=0,1,2, \ldots, d-1)$ are the settings of the appropriate phase shifters, for convenience we denote them as a $d$ dimensional vector $\vec{\phi}=\left(\phi^{0}, \phi^{1}, \phi^{2}, \ldots, \phi^{d-1}\right)$. For four-dimensional systems, $d=4$.

The quantum prediction for the probabilities of obtaining the outcome $(a, b, c)$ is then given as

$$
P\left(a_{i}=a, b_{j}=b, c_{k}=c\right)=\left|\left\langle a b c\left|U\left(\overrightarrow{\phi_{A}}\right) \otimes U\left(\overrightarrow{\phi_{B}}\right) \otimes U\left(\overrightarrow{\phi_{C}}\right)\right| \psi_{4}\right\rangle\right|^{2} .
$$

Thus the quantum analogue of the joint probability can be easily calculated

$$
\begin{aligned}
& P\left(a_{i}+b_{j}+c_{k}=r\right) \\
& =\frac{1}{16}\left(4+2 \cos \left(\varphi^{1}-\varphi^{0}+\frac{\pi}{2} r\right)+2 \cos \left(\varphi^{2}-\varphi^{0}+\pi r+2 \cos \left(\varphi^{2}-\varphi^{1}+\frac{\pi}{2} r\right)\right.\right. \\
& \left.+2 \cos \left(\varphi^{3}-\varphi^{0}+\frac{3 \pi}{2} r\right)+2 \cos \left(\varphi^{3}-\varphi^{1}+\pi r\right)+2 \cos \left(\varphi^{3}-\varphi^{2}+\frac{\pi}{2} r\right)\right),
\end{aligned}
$$

where $\varphi^{i}=\phi_{A}^{i}+\phi_{B}^{i}+\phi_{C}^{i},(i=0,1,2,3)$. In order to look for the maximal violation of the inequality, we choose the optimal settings as the following: $\vec{\phi}_{A 1}=\vec{\phi}_{B 1}=\vec{\phi}_{C 1}=\left(0, \frac{1}{3} \arccos \left(-\frac{1}{3}\right), \frac{1}{3} \arccos \left(-\frac{1}{3}\right)-\frac{\pi}{3}, \frac{\pi}{3}\right), \vec{\phi}_{A 2}=\vec{\phi}_{B 2}=$ $\vec{\phi}_{C 2}=\left(0, \frac{1}{3} \arcsin \frac{7}{9}, \frac{1}{3} \arcsin \frac{7}{9}+\frac{\pi}{6},-\frac{\pi}{6}\right)$. Numerical results show that for this choice, all the probability terms have definite values as listed in Table [1] Putting them into the left hand side of the inequality (5), we arrive at $2 \frac{1}{6}+3 \frac{2}{3}+3\left(3 \frac{1}{2}+3 \frac{1}{2}\right)+3\left(3 \frac{2}{3}+2 \frac{1}{6}\right)-2 \frac{1}{18}+5 \frac{8}{9}=\frac{68}{3}>12$.

In Ref. [16], a proposal was made to measure the strength of violation of local realism by the minimal amount of noise that must be added to the system in order to hide the non-classical character of the observed correlations. This is equivalent to a replacement of the pure state $|\psi\rangle\langle\psi|$ by the mixed state $\rho(F)$ of the form $\rho(F)=(1-F)|\psi\rangle\langle\psi|+$ $\frac{F}{56} I \otimes I \otimes I$, where $I$ is an identity matrix and $F(0 \leq F \leq 1)$ is the amount of noise present in the system. For $F=0$, local realistic description does not exist, whereas it does for $F=1$. Therefore, there exists some threshold value of $F$, denoted by $F_{t h r}$, such that for every $F \leq F_{t h r}$, local and realistic description does not exist. The threshold fidelity for the three four-level systems is determined by $\left(1-F_{t h r}\right) \frac{68}{3}=12$, namely $F_{t h r}=\frac{8}{17}=0.4706$.

Similarly, we propose a Bell inequality for three five-dimensional systems based on the Gedanken experiment:

$$
-2 P\left(a_{1}+b_{1}+c_{1}=0\right)+P\left(a_{1}+b_{1}+c_{1}=1\right)+P\left(a_{1}+b_{1}+c_{1}=4\right)
$$




\begin{tabular}{|c|c|c|c|}
\hline$p\left(a_{1}+b_{1}+c_{1}=0\right)$ & $p\left(a_{1}+b_{1}+c_{1}=1\right)$ & $p\left(a_{1}+b_{1}+c_{1}=2\right)$ & $p\left(a_{1}+b_{1}+c_{1}=3\right)$ \\
\hline 0 & $1 / 6$ & $2 / 3$ & $1 / 6$ \\
\hline$p\left(a_{1}+b_{1}+c_{2}=0\right)$ & $p\left(a_{1}+b_{1}+c_{2}=1\right)$ & $p\left(a_{1}+b_{1}+c_{2}=2\right)$ & $p\left(a_{1}+b_{1}+c_{2}=3\right)$ \\
\hline $1 / 2$ & 0 & $1 / 2$ & 0 \\
\hline$p\left(a_{1}+b_{2}+c_{1}=0\right)$ & $p\left(a_{1}+b_{2}+c_{1}=1\right)$ & $p\left(a_{1}+b_{2}+c_{1}=2\right)$ & $p\left(a_{1}+b_{2}+c_{1}=3\right)$ \\
\hline $1 / 2$ & 0 & $1 / 2$ & 0 \\
\hline$p\left(a_{2}+b_{1}+c_{1}=0\right)$ & $p\left(a_{2}+b_{1}+c_{1}=1\right)$ & $p\left(a_{2}+b_{1}+c_{1}=2\right)$ & $p\left(a_{2}+b_{1}+c_{1}=3\right)$ \\
\hline $1 / 2$ & 0 & $1 / 2$ & 0 \\
\hline$p\left(a_{1}+b_{2}+c_{2}=0\right)$ & $p\left(a_{1}+b_{2}+c_{2}=1\right)$ & $p\left(a_{1}+b_{2}+c_{2}=2\right)$ & $p\left(a_{1}+b_{2}+c_{2}=3\right)$ \\
\hline $2 / 3$ & $1 / 6$ & 0 & $1 / 6$ \\
\hline$p\left(a_{2}+b_{1}+c_{2}=0\right)$ & $p\left(a_{2}+b_{1}+c_{2}=1\right)$ & $p\left(a_{2}+b_{1}+c_{2}=2\right)$ & $p\left(a_{2}+b_{1}+c_{2}=3\right)$ \\
\hline $2 / 3$ & $1 / 6$ & 0 & $1 / 6$ \\
\hline$p\left(a_{2}+b_{2}+c_{1}=0\right)$ & $p\left(a_{2}+b_{2}+c_{1}=1\right)$ & $p\left(a_{2}+b_{2}+c_{1}=2\right)$ & $p\left(a_{2}+b_{2}+c_{1}=3\right)$ \\
\hline $2 / 3$ & $1 / 6$ & 0 & $1 / 6$ \\
\hline$p\left(a_{2}+b_{2}+c_{2}=0\right)$ & $p\left(a_{2}+b_{2}+c_{2}=1\right)$ & $p\left(a_{2}+b_{2}+c_{2}=2\right)$ & $p\left(a_{2}+b_{2}+c_{2}=3\right)$ \\
\hline $1 / 18$ & 0 & $1 / 18$ & $8 / 9$ \\
\hline
\end{tabular}

TABLE I: The values of the quantum joint probabilities in inequality (5) with appropriate angle settings.

$$
\begin{aligned}
& +P\left(a_{1}+b_{1}+c_{2}=0\right)-2 P\left(a_{1}+b_{1}+c_{2}=2\right)+P\left(a_{1}+b_{1}+c_{2}=4\right) \\
& +P\left(a_{1}+b_{2}+c_{1}=0\right)-2 P\left(a_{1}+b_{2}+c_{1}=2\right)+P\left(a_{1}+b_{2}+c_{1}=4\right) \\
& +P\left(a_{2}+b_{1}+c_{1}=0\right)-2 P\left(a_{2}+b_{1}+c_{1}=2\right)+P\left(a_{2}+b_{1}+c_{1}=4\right) \\
& +P\left(a_{1}+b_{2}+c_{2}=0\right)+P\left(a_{1}+b_{2}+c_{2}=3\right)-2 P\left(a_{1}+b_{2}+c_{2}=4\right) \\
& +P\left(a_{2}+b_{1}+c_{2}=0\right)+P\left(a_{2}+b_{1}+c_{2}=3\right)-2 P\left(a_{2}+b_{1}+c_{2}=4\right) \\
& +P\left(a_{2}+b_{2}+c_{1}=0\right)+P\left(a_{2}+b_{2}+c_{1}=3\right)-2 P\left(a_{2}+b_{2}+c_{1}=4\right) \\
& -2 P\left(a_{2}+b_{2}+c_{2}=1\right)+P\left(a_{2}+b_{2}+c_{2}=3\right)+P\left(a_{2}+b_{2}+c_{2}=4\right) \leq 4,
\end{aligned}
$$

which is satisfied by the local and realistic theories. By the way, the Bell inequality (10) is also tight [14].

Using specified quantum state and measurement, we calculate the maximum value that can be attained for the inequality (10). The considered state is a natural generalization of bipartite maximally entangled state to three five-level systems,

$$
\left|\psi_{5}\right\rangle=\frac{1}{\sqrt{5}}(|000\rangle+|111\rangle+|222\rangle+|333\rangle+|444\rangle) .
$$

The measurement is also based on unbiased symmetric multi-port beam splitter with $d=5$ and the quantum prediction for the probabilities of obtaining the outcome $(a, b, c)$ is then given as

$$
P\left(a_{i}=a, b_{j}=b, c_{k}=c\right)=\left|\left\langle a b c\left|U\left(\overrightarrow{\phi_{A}}\right) \otimes U\left(\overrightarrow{\phi_{B}}\right) \otimes U\left(\overrightarrow{\phi_{C}}\right)\right| \psi_{5}\right\rangle\right|^{2} .
$$

Thus the quantum analogue of the joint probability is given as

$$
\begin{aligned}
& P\left(a_{i}+b_{j}+c_{k}=r\right) \\
& =\frac{1}{25}\left(5+2 \cos \left(\varphi^{1}-\varphi^{0}+\frac{2 \pi}{5} r\right)+2 \cos \left(\varphi^{2}-\varphi^{0}+\frac{4 \pi}{5} r\right)+2 \cos \left(\varphi^{2}-\varphi^{1}+\frac{2 \pi}{5} r\right)\right. \\
& +2 \cos \left(\varphi^{3}-\varphi^{0}+\frac{6 \pi}{5} r\right)+2 \cos \left(\varphi^{3}-\varphi^{1}+\frac{4 \pi}{5} r\right)+2 \cos \left(\varphi^{3}-\varphi^{2}+\frac{2 \pi}{5} r\right) \\
& \left.+2 \cos \left(\varphi^{4}-\varphi^{0}+\frac{8 \pi}{5} r\right)+2 \cos \left(\varphi^{4}-\varphi^{1}+\frac{6 \pi}{5} r\right)+2 \cos \left(\varphi^{4}-\varphi^{2}+\frac{4 \pi}{5} r\right)+2 \cos \left(\varphi^{4}-\varphi^{3}+\frac{2 \pi}{5} r\right)\right),
\end{aligned}
$$

where $\varphi^{i}=\phi_{A}^{i}+\phi_{B}^{i}+\phi_{C}^{i}(i=0,1,2,3,4)$. Numerical results show that for the following angle settings $\vec{\phi}_{A 1}=\vec{\phi}_{B 1}=$ $\vec{\phi}_{C 1}=\left(0, \beta_{1}, \beta_{2},-\beta_{2},-\beta_{1}\right), \vec{\phi}_{A 2}=\vec{\phi}_{B 2}=\vec{\phi}_{C 2}=\left(0, \beta_{1}+\frac{\pi}{5}, \beta_{2}+\frac{2 \pi}{5},-\beta_{2}-\frac{2 \pi}{5},-\beta_{1}-\frac{\pi}{5}\right)$, where $\cos \left(3 \beta_{1}\right)-\cos \left(3 \beta_{2}\right)=\frac{1}{2}$, the maximum value of the left hand side of inequality (10) attained is 6.72216 . The threshold fidelity for the three five-level systems is determined by $\left(1-F_{t h r}\right) \times 6.72216=4$, namely $F_{t h r}=0.40495$. 
Given the above known Bell inequalities for three particles with $d=2,3,4,5$, it is worthy of noting that such tight inequalities exhibit perfect symmetries. One symmetry is that these Bell inequalities are symmetric under the permutations of subsystems $A, B$, and $C$. The second symmetry is that they can be expressed in a general form. Based on which, the structure of tight Bell inequalities is suggested as

$$
\frac{1}{d(d-1)} \sum_{i j k} \sum_{r=0}^{d-1} f_{d ; i j k}^{r} P\left(a_{i}+b_{j}+c_{k}=r\right) \leq 1,
$$

with $-1 \leq \frac{f_{d ; i j k}^{r}}{d(d-1)} \leq 1$. It is worthy of noting that the tight Bell inequalities for two qudits (i.e., the CGLMP inequality, see [5]) can be written in a similar form,

$$
\frac{1}{d-1} \sum_{i j} \sum_{r=0}^{d-1} f_{d ; i j}^{r} P\left(a_{i}+b_{j}=r\right) \leq 1,
$$

with $-1 \leq \frac{f_{d ; i j}^{r}}{d-1} \leq 1$. The coefficients $f_{d ; i j k}^{r}$ and $f_{d ; i j}^{r}$ are integers or half integers. Note that these inequalities are fulfilled with displacement of probabilities. In other words, for a known number $m$, where $m \leq d-1$, the above inequalities are still true for local realistic description by replacing $P\left(a_{i}+b_{j}+c_{k}=r\right)$ with $P\left(a_{i}+b_{j}+c_{k}=r+m\right)$, which is the third symmetry of the set of Bell inequalities for three particles.

\section{INTERESTING BELL INEQUALITIES OF THREE-QUBIT REDUCED FROM THOSE OF THREE-QUDIT}

In 1991 Gisin presented a theorem, which states that any pure entangled state of two particles violates a Bell inequality for two-particle correlation functions [9, 10]. Recent investigations show a surprising result that there exists a family of pure entangled $N$-qubit states that does not violate any Bell inequality for $N$-particle correlations for the case of a standard Bell experiment on $N$ qubits [17]. This family is the generalized GHZ states given by

$$
|\psi\rangle_{G H Z}=\cos \xi|0 \cdots 0\rangle+\sin \xi|1 \cdots 1\rangle
$$

with $0 \leq \xi \leq \pi / 4$. The usual GHZ states [18] are for $\xi=\pi / 4$. For a three-qubit system, whose corresponding generalized GHZ state reads $|\psi\rangle_{G H Z}=\cos \xi|000\rangle+\sin \xi|111\rangle$, it has been shown that for the region $\xi \in(0, \pi / 12]$, the inequalities given in [6] are not violated based on the standard Bell experiment. Recently, we developed a three-qubit Bell inequality which is a solution to such a problem. That is all pure entangled states of three qubits violates the Bell inequality given in [12]. Indeed Bell inequalities are sensitive to the presence of noise and above a certain amount of noise the Bell inequalities will cease to be violated by a quantum system. However, it seems that the inequality in Ref. [12] is not good enough to the resistance of noise. For the three-qubit GHZ state, the threshold visibility is $V_{G H Z}=4 \sqrt{3} / 9=0.7698$ and for the $\mathrm{W}$ state, the threshold visibility is $V_{W}=0.7312$. Tne inequality in Ref. [12] can be derived from three-qutrit Bell inequality (3). Actually any Bell inequality for tripartite $d(d>2)$-level systems may reduce to a Bell inequality for three qubits when one considers only two outcomes of measurement.

Here we present a new Bell inequality for three-qubit systems which is reduced from inequality (5)

$$
\begin{aligned}
& 3 P\left(a_{1}+b_{1}+c_{1}=0\right)+P\left(a_{1}+b_{1}+c_{1}=1\right)-5 P\left(a_{1}+b_{1}+c_{1}=2\right)+P\left(a_{1}+b_{1}+c_{1}=3\right) \\
& +3 P\left(a_{1}+b_{1}+c_{2}=0\right)+P\left(a_{1}+b_{1}+c_{2}=1\right)+3 P\left(a_{1}+b_{1}+c_{2}=2\right)-7 P\left(a_{1}+b_{1}+c_{2}=3\right) \\
& +3 P\left(a_{1}+b_{2}+c_{1}=0\right)+P\left(a_{1}+b_{2}+c_{1}=1\right)+3 P\left(a_{1}+b_{2}+c_{1}=2\right)-7 P\left(a_{1}+b_{2}+c_{1}=3\right) \\
& +3 P\left(a_{2}+b_{1}+c_{1}=0\right)+P\left(a_{2}+b_{1}+c_{1}=1\right)+3 P\left(a_{2}+b_{1}+c_{1}=2\right)-7 P\left(a_{2}+b_{1}+c_{1}=3\right) \\
& -5 P\left(a_{1}+b_{2}+c_{2}=0\right)+P\left(a_{1}+b_{2}+c_{2}=1\right)+3 P\left(a_{1}+b_{2}+c_{2}=2\right)+P\left(a_{1}+b_{2}+c_{2}=3\right) \\
& -5 P\left(a_{2}+b_{1}+c_{2}=0\right)+P\left(a_{2}+b_{1}+c_{2}=1\right)+3 P\left(a_{2}+b_{1}+c_{2}=2\right)+P\left(a_{2}+b_{1}+c_{2}=3\right) \\
& -5 P\left(a_{2}+b_{2}+c_{1}=0\right)+P\left(a_{2}+b_{2}+c_{1}=1\right)+3 P\left(a_{2}+b_{2}+c_{1}=2\right)+P\left(a_{2}+b_{2}+c_{1}=3\right) \\
& \left.-P\left(a_{2}+b_{2}+c_{2}=0\right)+5 P\left(a_{2}+b_{2}+c_{2}=1\right)-P\left(a_{2}+b_{2}+c_{2}=2\right)-3 P\left(a_{2}+b_{2}+c_{2}=3\right)\right) \leq 12 .
\end{aligned}
$$

The inequality can be expressed in terms of correlation functions

$$
\begin{aligned}
& -E\left(A_{1} B_{1} C_{1}\right)+E\left(A_{1} B_{1} C_{2}\right)+E\left(A_{1} B_{2} C_{1}\right)+E\left(A_{2} B_{1} C_{1}\right)-E\left(A_{2} B_{2} C_{2}\right) \\
& -E\left(A_{1} B_{2}\right)-E\left(A_{2} B_{1}\right)-E\left(A_{2} B_{2}\right)-E\left(A_{1} C_{2}\right)-E\left(A_{2} C_{1}\right)-E\left(A_{2} C_{2}\right) \\
& -E\left(B_{1} C_{2}\right)-E\left(B_{2} C_{1}\right)-E\left(B_{2} C_{2}\right)+E\left(A_{1}\right)+E\left(B_{1}\right)+E\left(C_{1}\right) \leq 3 .
\end{aligned}
$$




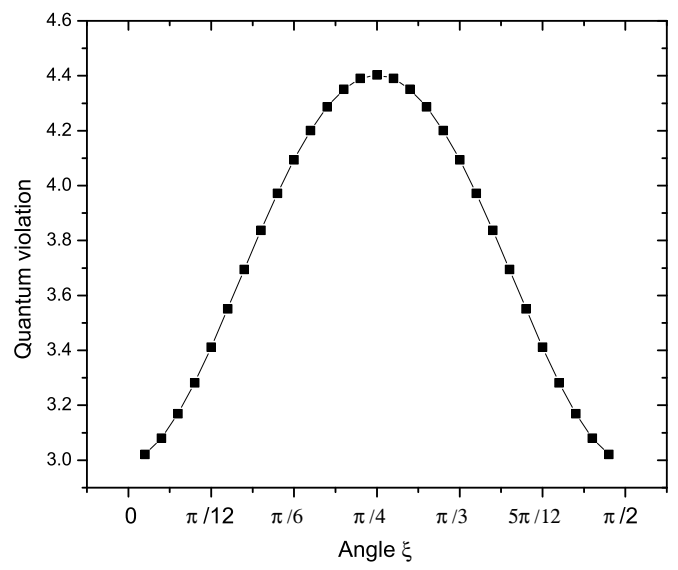

FIG. 1: Numerical results for the generalized GHZ states $\left|\psi_{2}\right\rangle_{\mathrm{GHZ}}=\cos \xi|000\rangle+\sin \xi|111\rangle$, which violate the inequality (18) except $\xi=0, \pi / 2$.

The above inequality (18) includes the terms of single correlation functions, it is symmetric under the permutations of $A_{j}, B_{j}$ and $C_{j}$. Quantum mechanically, the above inequality is violated by all pure entangled states of three qubits. Pure states of three qubits constitute a five-parameter family, with equivalence up to local unitary transformations. This family has the following representation [19]

$$
\begin{aligned}
|\psi\rangle= & \sqrt{\mu_{0}}|000\rangle+\sqrt{\mu_{1}} e^{i \phi}|100\rangle+\sqrt{\mu_{2}}|101\rangle \\
& +\sqrt{\mu_{3}}|110\rangle+\sqrt{\mu_{4}}|111\rangle
\end{aligned}
$$

with $\mu_{i} \geq 0, \sum_{i} \mu_{i}=1$, and $0 \leq \phi \leq \pi$. Numerical results show that this Bell inequality for probabilities is violated by all pure entangled states of three-qubit systems. However, no analytical proof of the conclusion has been given. In the following, some special cases will be given to show the inequality (18) is violated by all pure entangled states. The first quantum state considered is generalized GHZ state $\left|\psi_{2}\right\rangle_{\mathrm{GHZ}}=\cos \xi|000\rangle+\sin \xi|111\rangle$. The inequality (18) is violated by the generalized GHZ states for the whole region except $\xi=0, \pi / 2$. For the GHZ state with $\xi=\pi / 4$, the quantum violation reaches its maximum value 4.40367. The variation of the violation with $\xi$ is shown in Fig. 1, Another state considered is generalized $\mathrm{W}$ state $|\psi\rangle_{W}=\sin \beta \cos \xi|100\rangle+\sin \beta \sin \xi|010\rangle+\cos \beta|001\rangle$. By fixing the value of $\beta$, quantum violation of the inequality (18) varies with $\xi$ (see Fig. 22). The inequality (18) is violated by the generalized W states except the cases with $\beta=\frac{\pi}{2}, \xi=0$ and $\xi=\frac{\pi}{2}$. The states in these cases are direct-product states which do not violated any Bell inequality. For the standard W state, quantum violation of the inequality (18) approaches 4.54086 .

Hence the inequality (18) is also one candidate to generalize the theorem of Gisin to three-qubit systems. One of the features of the new inequality for three qubits is that it is highly resistant to noise. The inequality (18) is violated by the generalized GHZ state $\left|\psi_{2}\right\rangle_{G H Z}=\cos \xi|000\rangle+\sin \xi|111\rangle$ for the whole region, the threshold visibility is $V_{t h r}^{G H Z}=0.68125$. The inequality (18) is also violated by the $\mathrm{W}$ state, the threshold visibility is $V_{t h r}^{W}=0.660668$. We plot the variation of quantum violation for the generalized GHZ states with angle $\xi$ for inequality (18) and inequality given in Ref. [12], see Fig. 3. In plotting the figure, we reform the expressions of these two inequalities as

$$
\begin{array}{cl}
\frac{1}{4}[ & Q\left(A_{1} B_{1} C_{1}\right)-Q\left(A_{1} B_{2} C_{2}\right)-Q\left(A_{2} B_{1} C_{2}\right)-Q\left(A_{2} B_{2} C_{1}\right)+2 Q\left(A_{2} B_{2} C_{2}\right) \\
& -Q\left(A_{1} B_{1}\right)-Q\left(A_{1} B_{2}\right)-Q\left(A_{2} B_{1}\right)-Q\left(A_{2} B_{2}\right)+Q\left(A_{1} C_{1}\right)+Q\left(A_{1} C_{2}\right) \\
+ & \left.Q\left(A_{2} C_{1}\right)+Q\left(A_{2} C_{2}\right)+Q\left(B_{1} C_{1}\right)+Q\left(B_{1} C_{2}\right)+Q\left(B_{2} C_{1}\right)+Q\left(B_{2} C_{2}\right)\right] \leq 1, \\
\frac{1}{3}[ & -Q\left(A_{1} B_{1} C_{1}\right)+Q\left(A_{1} B_{1} C_{2}\right)+Q\left(A_{1} B_{2} C_{1}\right)+Q\left(A_{2} B_{1} C_{1}\right)-Q\left(A_{2} B_{2} C_{2}\right) \\
& -Q\left(A_{1} B_{2}\right)-Q\left(A_{2} B_{1}\right)-Q\left(A_{2} B_{2}\right)-Q\left(A_{1} C_{2}\right)-Q\left(A_{2} C_{1}\right)-Q\left(A_{2} C_{2}\right) \\
& \left.-Q\left(B_{1} C_{2}\right)-Q\left(B_{2} C_{1}\right)-Q\left(B_{2} C_{2}\right)+Q\left(A_{1}\right)+Q\left(B_{1}\right)+Q\left(C_{1}\right)\right] \leq 1,
\end{array}
$$




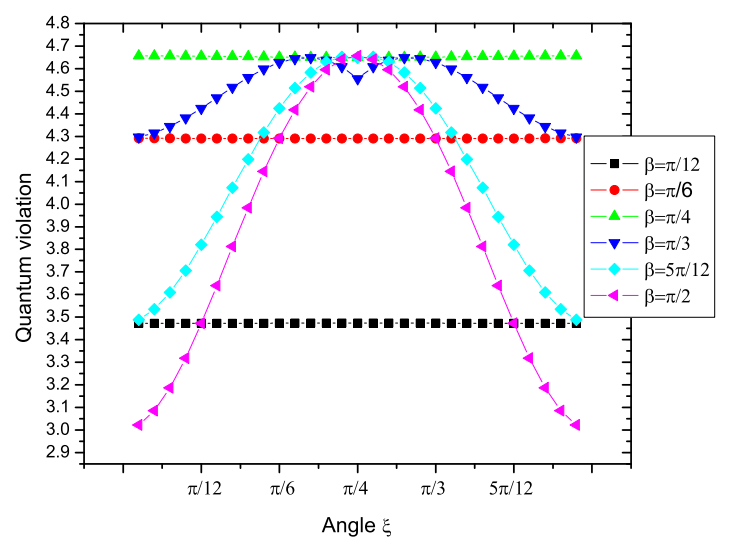

FIG. 2: Numerical results for the generalized W states $|\psi\rangle_{W}=\sin \beta \cos \xi|100\rangle+\sin \beta \sin \xi|010\rangle+\cos \beta|001\rangle$ which violate the inequality (18) for different $\xi$ and $\beta$. Here the cases $\beta=\pi / 12, \pi / 6, \pi / 4, \pi / 3,5 \pi / 12$, and $\pi / 2$ are considered.

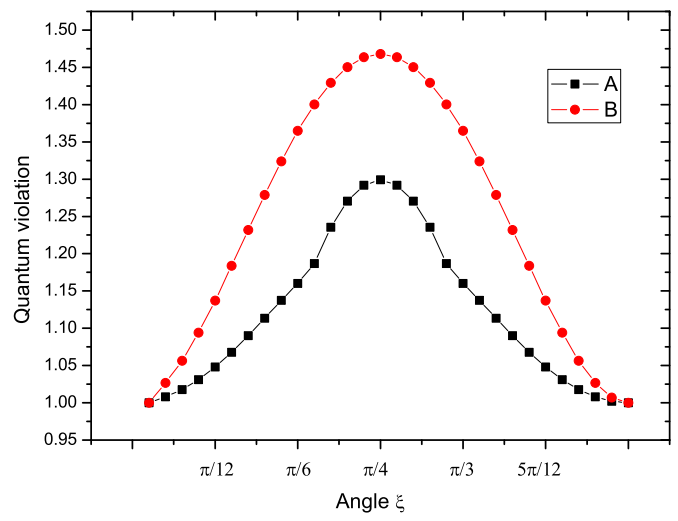

FIG. 3: Violation of two Bell inequalities for the generalized GHZ state of three qubits with different value of $\xi$, where curve $\mathrm{A}$ is for inequality given in Ref. [12] and curve B is for a new inequality (18).

respectively. By the reformation, the violation degrees of the two inequalities can be compared directly. Comparing the results of the inequality given in Ref. [12], the new inequality (18) is indeed more resistant to noise. It seems that we could derive some new three-qubit Bell inequalities, which would be more highly resistance to noise, if we get other Bell inequalities of tripartite $d$-dimensional $(d>4)$ quantum systems.

When setting $C_{1}=-1, C_{2}=1$, the inequality (18) reduces directly to the CHSH inequality for two-qubit

$$
E\left(A_{1} B_{1}\right)-E\left(A_{1} B_{2}\right)-E\left(A_{2} B_{1}\right)-E\left(A_{2} B_{2}\right) \leq 2,
$$

which is equivalent to

$$
E\left(A_{1} B_{1}\right)+E\left(A_{1} B_{2}\right)+E\left(A_{2} B_{1}\right)-E\left(A_{2} B_{2}\right) \leq 2 .
$$

Starting from the Bell inequality for three five-level systems, another three-qubit Bell inequality can be obtained as

$$
\begin{aligned}
& P\left(a_{1}+b_{1}+c_{1}=1\right)-2 P\left(a_{1}+b_{1}+c_{1}=2\right)+P\left(a_{1}+b_{1}+c_{1}=3\right)+P\left(a_{1}+b_{1}+c_{2}=1\right)+P\left(a_{1}+b_{1}+c_{2}=2\right) \\
& +P\left(a_{1}+b_{2}+c_{1}=1\right)+P\left(a_{1}+b_{2}+c_{1}=2\right)+P\left(a_{2}+b_{1}+c_{1}=1\right)+P\left(a_{2}+b_{1}+c_{1}=2\right) \\
& +P\left(a_{1}+b_{2}+c_{2}=0\right)-2 P\left(a_{1}+b_{2}+c_{2}=1\right)+P\left(a_{1}+b_{2}+c_{2}=2\right)+P\left(a_{2}+b_{1}+c_{2}=0\right) \\
& -2 P\left(a_{2}+b_{1}+c_{2}=1\right)+P\left(a_{2}+b_{1}+c_{2}=2\right)+P\left(a_{2}+b_{2}+c_{1}=0\right)-2 P\left(a_{2}+b_{2}+c_{1}=1\right) \\
& +P\left(a_{2}+b_{2}+c_{1}=2\right)+P\left(a_{2}+b_{2}+c_{2}=0\right)+P\left(a_{2}+b_{2}+c_{2}=1\right)-2 P\left(a_{2}+b_{2}+c_{2}=3\right) \leq 4 .
\end{aligned}
$$


The above inequality is just the Mermin inequality when it is expressed in terms of correlation functions:

$$
-Q_{111}+Q_{122}+Q_{212}+Q_{221} \leq 2
$$

\section{SUMMARY}

To summarize, we have presented the tight Bell inequalities expressed by probabilities for three four- and fivedimensional systems. The tight structure of Bell inequalities for three $d$-dimensional systems (qudits) is also proposed. Moreover, a new Bell inequality for three qubits is derived (or say, reduced) from the inequality for three four-level systems. The inequality (18) is violated by any pure three-qubit entangled state, and it is more resistant to noise compared with the one given in Ref. [12]. Furthermore, the tight Mermin inequality for thre-qubit can be obtained by reducing the Bell inequality for three five-level systems [see Eq. (10) and Eq. (22)].

ACKNOWLEDGMENTS The authors thank A. Acin for many useful discussions. This work was supported by NUS research Grant No. WBS: R-144-000-123-112. J.L.C acknowledges financial supports from NSF of China (Grant No. 10605013), Program for New Century Excellent Talents in University, and the Project-sponsored by SRF for ROCS, SEM. C.F.W. acknowledges financial support from Singapore Millennium Foundation.

[1] J. S. Bell, Physics (Long Island City, N.Y.) 1, 195 (1964).

[2] J. Clauser, M. Horne, A. Shimony, and R. Holt, Phys. Rev. Lett. 23, 880 (1969).

[3] A. K. Ekert, Phys. Rev. Lett. 67, 661 (1991).

[4] A. Acín, N. Brunner, N. Gisin, S. Massar, S. Pironio, and V. Scarani, Phys. Rev. Lett. 98, 230501 (2007).

[5] D. Collins, N. Gisin, N. Linden, S. Massar, and S. Popescu, Phys. Rev. Lett. 88, 040404 (2002).

[6] R. F. Werner and M. M. Wolf, Phys. Rev. A 64, 032112 (2001); M. Żukowski and Č. Brukner, Phys. Rev. Lett. 88, 210401 (2002).

[7] A. Acín, J. L. Chen, N. Gisin, D. Kaszlikowski, L. C. Kwek, C. H. Oh, and M. Żukowski, Phys. Rev. Lett. 92, 250404 (2004).

[8] D. Kaszlikowski and M. Żukowski, Phys. Rev. A 66, 042107 (2002); N. J. Cerf, S. Massar, and S. Pironio, Phys. Rev. Lett. 89, 080402 (2002).

[9] N. Gisin, Phys. Lett. A 154, 201 (1991); N. Gisin and A. Peres, Phys. Lett. A 162, $15-17$ (1992).

[10] S. Popescu and D. Rohrlich, Phys. Lett. A 166, 293 (1992).

[11] M. Żukowski, Č. Brukner, W. Laskowski, and M. Wiesniak, Phys. Rev. Lett. 88, 210402 (2002).

[12] J. L. Chen, C. F. Wu, L. C. Kwek, and C.H . Oh, Phys. Rev. Lett. 93, 140407 (2004).

[13] N. D. Mermin, Phys. Rev. Lett. 65, 1838 (1990).

[14] Private communication with A. Acin, who has performed the culculation and proved that the Bell inequalities (5) and (10) were tight.

[15] M. Żukowski, Z. Zeilinger, and M. A. Horne, Phys. Rev. A 55, 2564 (1997).

[16] D. Kaszlikowski, P. Gnaciński, M. Żukowski, W. Miklaszewski, and A. Zeilinger, Phys. Rev. Lett. 85, 4418 (2000).

[17] V. Scarani and N. Gisin, J. Phys. A 346043 (2001).

[18] D. M. Greenberger, M. Horne, A. Shimony, and A. Zeilinger, Am. J. Phys. 58, 1131 (1990).

[19] A. Acín, A. Andrianov, L. Costa, E. Jané, J. I. Latorre, and R. Tarrach, Phys. Rev. Lett. 85, 1560 (2000). 